\title{
Exact analysis of the effects of sampling of the scalar diffraction field
}

\author{
Levent Onural \\ Department of Electrical and Electronics Engineering, Bilkent University, TR-06800 Ankara, Turkey
}

Received June 8, 2006; accepted July 28, 2006;

posted September 7, 2006 (Doc. ID 71699); published January 10, 2007

\begin{abstract}
If the sampled diffraction pattern due to a planar object is used to reconstruct the object pattern by backpropagation, the obtained pattern is no longer the same as the original. The effect of such sampling on the reconstruction is analyzed. The formulation uses the plane-wave expansion, and therefore the provided solution is exact for wave propagation in media where scalar wave propagation is valid. In contrast to the sampling effects under the Fresnel approximation, the exact solution indicates that there are no modulated replicas of the original object in the reconstructed pattern. Rather, the distortion is in the form of modulated, translated, and dispersed versions of the original. (C) 2007 Optical Society of America
\end{abstract}

OCIS codes: $050.1940,150.1960$.

\section{INTRODUCTION}

Reconstruction of underlying objects from their captured diffraction patterns, or holograms in more practical cases, by digital means is an attractive common practice. ${ }^{1-7}$ The associated inevitable sampling (discretization) of the diffraction field during the initial phase of subsequent digital processing requires care. ${ }^{8-12}$ As in many other applications, the band-limited sampling and the associated sinc interpolation are also commonly applied to diffraction. However, it is known that such a general approach, without taking into consideration the other properties and restrictions due to the nature of the diffraction and the objects, is bound to yield extremely inefficient results by producing an unnecessarily large amount of samples. For example, space-limited (thus non-band-limited) objects can be fully recovered from their Fresnel transforms, which are sampled rather sparsely. ${ }^{8,13}$ It is shown that the sampling of the Fresnel diffraction field results in modulated and translated replicas of the original at periodic locations. Therefore, a finite-extent (space-limited) object can be fully recovered by cutting away the undesired modulated replicas; the sampling rate determines the locations of the replicas: higher sampling rates generate farther translated modulated replicas of the object. ${ }^{8}$

It is noticed by Coupland ${ }^{14}$ that when the Fresnel approximation is no longer valid the sampling of the diffraction field does not create visible replicas in the reconstruction. Coupland got experimental results and provided some theoretical explanation for this phenomenon. Here in this paper, a rigorous proof for the above-mentioned observation and the exact analytical form of the introduced distortion due to the sampling are presented. A review of plane-wave decomposition formulation of diffraction is included in Section 2 for the sake of clarity and completeness of the subsequent sections.

\section{REVIEW OF PLANE-WAVE DECOMPOSITION FORMULATION OF DIFFRACTION}

Even though there is plenty of good literature on diffraction (see, for example, Goodman ${ }^{15}$ or Born and Wolf ${ }^{16}$ ), it may be useful to provide a brief review both to establish the notation and to provide a better basis for the rest of the paper. We concentrate on the plane-wave decomposition (angular spectrum representation) approach. ${ }^{15-18}$

For the scalar case, the plane-wave decomposition approach is exact, and simple and yields efficient computational algorithms. Briefly, a single 3-D plane wave $\exp \left(j \mathbf{k}^{T} \mathbf{x}\right)$ is a solution of the Helmholtz equation. Therefore, a superposition of plane waves,

$$
\psi(\mathbf{x})=\int B(\mathbf{k}) \exp \left(j \mathbf{k}^{T} \mathbf{x}\right) \mathrm{d} \mathbf{k},
$$

is also a solution and gives the 3-D field. The integration is over a set of k's that is determined by constraints associated with the physics of a given problem, where $\mathbf{k}$ $=\left[\begin{array}{lll}k_{x} & k_{y} & k_{z}\end{array}\right]^{T}$ and $\mathbf{x}=\left[\begin{array}{lll}x & y & z\end{array}\right]^{T}$.

If we restrict the wavenumber (the $3-\mathrm{D}$ spatial frequency) $\mathbf{k}$, such that $|\mathbf{k}|=k=2 \pi / \lambda$, where $\lambda$ is the wavelength to represent the monochromatic propagating waves, the integral becomes a surface integral over the $|\mathbf{k}|=2 \pi / \lambda$ (Ewald) sphere. ${ }^{19}$ The conventional diffraction problem between two parallel planes can be solved by intersecting the 3-D field of Eq. (1) by the two planes. ${ }^{20}$ As a consequence of the monochromatic propagating wave constraint, the variable $\mathbf{k}$ can be represented by two variables (directional components) $k_{x}$ and $k_{y}$, since $k_{z}=\left(k^{2}\right.$ $\left.-k_{x}^{2}-k_{y}^{2}\right)^{1 / 2}$. We define the 2 -D vector $\boldsymbol{k}$ to be equal to $\left[\begin{array}{ll}k_{x} & k_{y}\end{array}\right]^{T}$ to ease the notation. Similarly, $\boldsymbol{x}$ represents the 2 -D space variables $\left[\begin{array}{ll}x & y\end{array}\right]^{T}$. A positive $k_{z}$ represents 
propagation in the positive $z$ direction and vice versa. Therefore, it may be convenient to convert the integration measure dk of Eq. (1) into $\mathrm{d} \boldsymbol{k}=\mathrm{d} k_{x} \mathrm{~d} k_{y}$ and rewrite the integral as

$$
\psi(\mathbf{x})=\int_{k_{x}^{2}+k_{y}^{2} \leqslant k^{2}} A(\boldsymbol{k}) \exp \left(j \mathbf{k}^{T} \mathbf{x}\right) \mathrm{d} \boldsymbol{k},
$$

where, due to change of variables of the integration, the relation between the amplitude of the plane-wave components $B(\mathbf{k})$ and $A(\boldsymbol{k})$ is $^{19}$

$$
A(\boldsymbol{k})=B(\mathbf{k}) \frac{k}{\left(k^{2}-\boldsymbol{k}^{T} \boldsymbol{k}\right)^{1 / 2}} ;
$$

note that $B(\cdot)$ and $A(\cdot)$ are 3-D and 2-D functions, respectively. Choosing the two parallel planes as the $z=0$ (object) plane and $z=Z$ (diffraction) plane, we see that, for each plane-wave component,

$$
\begin{aligned}
& \left.A(\boldsymbol{k}) \exp \left(j \boldsymbol{k}^{T} \mathbf{x}\right)\right|_{z=0}=A(\boldsymbol{k}) \exp \left[j \boldsymbol{k}^{T} \mathbf{x}\right], \\
& \left.A(\boldsymbol{k}) \exp \left(j \boldsymbol{k}^{T} \mathbf{x}\right)\right|_{z=Z}=A(\boldsymbol{k}) \exp \left[j \boldsymbol{k}^{T} \mathbf{x}\right] \exp \left[j \phi_{Z}(\boldsymbol{k})\right],
\end{aligned}
$$

where $\phi_{Z}(\boldsymbol{k})$ is the phase shift due to the angle of propagation of the plane wave and linearly increases with the distance variable $Z$, as

$$
\phi_{Z}(\boldsymbol{k})=Z\left(k^{2}-\boldsymbol{k}^{T} \boldsymbol{k}\right)^{1 / 2},
$$

for a given $\boldsymbol{k}$. Therefore, the superposition of all such monochromatic propagating waves, over all $\boldsymbol{k}$ that fall into the disk $\left(k^{2}-\boldsymbol{k}^{T} \boldsymbol{k}\right) \geqslant 0$, gives us the object pattern at the $z=0$ plane and the diffraction pattern at the $z=Z$ plane, as

$$
\begin{aligned}
& \psi_{0}(\boldsymbol{x}) \triangleq \psi(x, y, 0)=\int A(\boldsymbol{k}) \exp \left[j \boldsymbol{k}^{T} \boldsymbol{x}\right] \mathrm{d} \boldsymbol{k}, \\
& \psi_{Z}(\boldsymbol{x}) \triangleq \psi(x, y, Z)=\int A(\boldsymbol{k}) \exp \left[j \boldsymbol{k}^{T} \boldsymbol{x}\right] \\
& \times \exp \left[j Z\left(k^{2}-\boldsymbol{k}^{T} \boldsymbol{k}\right)^{1 / 2}\right] \mathrm{d} \boldsymbol{k} .
\end{aligned}
$$

Please note that all the superpositions presented in expressions (1), (2), (7), and (8) are either 3-D or 2-D Fourier expansions for the corresponding diffraction patterns (which, of course, include the object pattern, as well). From expression (7), we see that

$$
4 \pi^{2} A(\boldsymbol{k})=\mathcal{F}\{\psi(\boldsymbol{x})\},
$$

and, from expression (8), we get the result

$$
\Psi_{Z}(\boldsymbol{k})=\mathcal{F}^{-1}\left\{4 \pi^{2} A(\boldsymbol{k}) H_{Z}(\boldsymbol{k})\right\},
$$

where

$$
\begin{aligned}
H_{Z}(\boldsymbol{k}) & =H_{Z}\left(k_{x}, k_{y}\right) \triangleq \exp \left[j Z\left(k^{2}-\boldsymbol{k}^{T} \boldsymbol{k}\right)^{1 / 2}\right] \\
& =\exp \left[j Z\left(k^{2}-k_{x}^{2}-k_{y}^{2}\right)^{1 / 2}\right] .
\end{aligned}
$$

Therefore, expression (8) [or, equivalently, Eq. (10)] indicates the well-known result that the diffraction pattern due to propagating waves, over a plane parallel to the given planar object pattern, at a distance $Z$, is the output of a linear shift-invariant system whose transfer function is $H_{Z}(\boldsymbol{k})$, which has a unity magnitude within its passband. The impulse response of this linear shift-invariant system, which is the inverse Fourier transform of $H_{Z}(\boldsymbol{k})$, is $^{21,22}$

$$
\begin{aligned}
h_{Z}(\boldsymbol{x}) & =h_{Z}(x, y) \\
& =\mathcal{F}^{-1}\left\{H_{Z}(\boldsymbol{k})\right\}=-\frac{1}{2 \pi} \frac{\partial}{\partial Z} \frac{\exp \left[j k\left(x^{2}+y^{2}+Z^{2}\right)^{1 / 2}\right]}{\left(x^{2}+y^{2}+Z^{2}\right)^{1 / 2}} \\
& =-\frac{1}{2 \pi} \frac{\partial}{\partial Z} \frac{\exp \left[j k\left(\boldsymbol{x}^{T} \boldsymbol{x}+Z^{2}\right)^{1 / 2}\right]}{\left(\boldsymbol{x}^{T} \boldsymbol{x}+Z^{2}\right)^{1 / 2}} \\
& =-\frac{1}{2 \pi} \frac{\partial}{\partial Z} \frac{\exp (j k r)}{r} .
\end{aligned}
$$

If the diffraction due to illumination of a physical mask is considered, evanescent wave components should also be included in the discussion. Indeed, the Fourier transform relation between expressions (12) and (11) includes both propagating $\left(\boldsymbol{k}^{T} \boldsymbol{k}=k_{x}^{2}+k_{y}^{2} \leqslant k_{z}^{2}\right)$ and evanescent $\left(\boldsymbol{k}^{T} \boldsymbol{k}=k_{x}^{2}\right.$ $+k_{y}^{2}>k_{z}^{2}$ ) components. ${ }^{21}$ However, if the physical mask is a spatially low-pass function with a 2 -D passband $k_{x}^{2}+k_{y}^{2}$ $\leqslant k_{z}^{2}$ or if the mask is simply obtained by intersecting the $3-\mathrm{D}$ field due to propagating waves with the $z=0$ plane, then there are no evanescent components. Therefore, the Rayleigh-Sommerfeld diffraction and the plane-wave decomposition are equivalent, as also shown by Sherman. ${ }^{21}$ As a conclusion, we can say that the RayleighSommerfeld diffraction whose convolution kernel is given by Eq. (12) is the exact solution to the scalar diffraction problem. Furthermore, as a consequence of the Fourier transform relations given by Eqs. (9) and (10), the computational burden of this exact solution in simulations is comparable to that of the Fresnel case, and therefore, considering the accuracy and the broader applicability, one may prefer the Rayleigh-Sommerfeld diffraction formulation (or the equivalent plane-wave decomposition) over other approximate diffraction formulations. Both the impulse response [Eq. (12)] and its Fourier transform [expression (11)] corresponding to the linear shift-invariant system representing the exact scalar diffraction between parallel planes are analytically known, as presented above, anyway.

The 3-D field over the space $z \geqslant 0$ may be physically generated by illuminating the 2 -D object mask by a single plane wave propagating along the $z$ axis. Similarly, if a complex-valued mask records the diffraction pattern at $z=Z$ and if this mask is illuminated by a single plane wave propagating along the $-z$ direction, the $3-\mathrm{D}$ field is obtained physically for the space $z \leqslant Z$; this is commonly called the reconstruction. Reconstruction refers to the process of creating $\psi(\mathbf{x})$ [and thus $\psi_{0}(\boldsymbol{x})$ ] from $\psi_{Z}(\boldsymbol{x})$, and that can be accomplished by implementing the inverse system by physical or computational means. Mathematically, the reconstruction is equivalent to

$$
\psi_{0}(\boldsymbol{x})=\mathcal{F}^{-1}\left\{\Psi_{Z}(\boldsymbol{k}) H_{-Z}(\boldsymbol{k})\right\}=\psi_{Z}(\boldsymbol{x}) * * h_{-Z}(\boldsymbol{x})
$$

as a consequence of the properties

$$
H_{Z}(\boldsymbol{k}) H_{-Z}(\boldsymbol{k})=1
$$




$$
h_{Z}(\boldsymbol{x}) * * h_{-Z}(\boldsymbol{x})=\delta(\boldsymbol{x}),
$$

where $* *$ denotes 2-D convolution and $h_{-Z}(\boldsymbol{x})$ is the convolution kernel for the reverse propagation that is obtained by replacing $Z$ by $-Z$ in $h_{Z}(\boldsymbol{x})$. The function $\delta(\boldsymbol{x})$ is the 2-D impulse function.

\section{EFFECT OF SAMPLING-EXACT ANALYSIS}

Let us consider the sampling of the 2-D scalar diffraction pattern, $\psi_{Z}(\boldsymbol{x})$ over the plane $z=Z$, by a 2 -D lattice. This corresponds to the multiplication of the pattern by a regular (periodic) lattice of impulse functions:

$$
\psi_{Z s}(\boldsymbol{x}) \triangleq \psi_{Z}(\boldsymbol{x}) \sum_{\boldsymbol{n}} \delta(\boldsymbol{x}-\boldsymbol{V n})=\sum_{\boldsymbol{n}} \psi_{Z}(\boldsymbol{V} \boldsymbol{n}) \delta(\boldsymbol{x}-\boldsymbol{V n}),
$$

where $\psi_{Z s}(\boldsymbol{x})$ is the 2-D discrete (sampled) diffraction field, $\boldsymbol{V}$ is the $2-\mathrm{D}$ sampling matrix, $\delta(\boldsymbol{x})$ is the $2-\mathrm{D}$ impulse function, and $\boldsymbol{n}=\left[\begin{array}{ll}n_{1} & n_{2}\end{array}\right]^{T}$ is a vector of integers. We label the sampling function as

$$
p(\boldsymbol{x})=\sum_{\boldsymbol{n}} \delta(\boldsymbol{x}-\boldsymbol{V n}) .
$$

Using the Fourier relations due to sampling, ${ }^{8,23}$ we get

$$
\Psi_{Z s}(\boldsymbol{k})=\mathcal{F}\left\{\psi_{Z s}(\boldsymbol{x})\right\}=\frac{1}{|\operatorname{det} \boldsymbol{V}|} \sum_{\boldsymbol{m}} \Psi_{Z}(\boldsymbol{k}-\boldsymbol{U} \boldsymbol{m}),
$$

where $\boldsymbol{U}=2 \pi \boldsymbol{V}^{-T}$ and $\boldsymbol{m}$ is a $2-\mathrm{D}$ vector of integers.

Therefore, the Fourier transform, $\Psi_{R}(\boldsymbol{k})$, of the reconstruction from the sampled diffraction pattern is ${ }^{8}$

$$
\begin{aligned}
\Psi_{R}(\boldsymbol{k}) & =\Psi_{Z s}(\boldsymbol{k}) H_{-Z}(\boldsymbol{k})=\frac{1}{|\operatorname{det} \boldsymbol{V}|} \sum_{\boldsymbol{m}} \Psi_{Z}(\boldsymbol{k}-\boldsymbol{U m}) H_{-Z}(\boldsymbol{k}) \\
& =\frac{1}{|\operatorname{det} \boldsymbol{V}|} \sum_{\boldsymbol{m}} 4 \pi^{2} A(\boldsymbol{k}-\boldsymbol{U} \boldsymbol{m}) H_{Z}(\boldsymbol{k}-\boldsymbol{U} \boldsymbol{m}) H_{-Z}(\boldsymbol{k}) .
\end{aligned}
$$

We can analytically find $H_{Z}(\boldsymbol{k}-\boldsymbol{U m}) H_{-Z}(\boldsymbol{k})$ of Eq. (19) as

$$
\begin{aligned}
H_{Z}(\boldsymbol{k}-\boldsymbol{U m}) H_{-Z}(\boldsymbol{k})= & \exp \left\{j Z\left[k^{2}-(\boldsymbol{k}-\boldsymbol{U m})^{T}(\boldsymbol{k}-\boldsymbol{U m})\right]^{1 / 2}\right\} \\
& \times \exp \left\{-j Z\left[k^{2}-\boldsymbol{k}^{T} \boldsymbol{k}\right]^{1 / 2}\right\},
\end{aligned}
$$

and therefore Eq. (19) becomes

$$
\begin{aligned}
\Psi_{R}(\boldsymbol{k})= & \frac{4 \pi^{2}}{|\operatorname{det} \boldsymbol{V}|} \sum_{\boldsymbol{m}} A(\boldsymbol{k}-\boldsymbol{U m}) \exp \left(j Z \left\{\left[k^{2}-(\boldsymbol{k}-\boldsymbol{U m})^{T}\right.\right.\right. \\
& \left.\left.\times(\boldsymbol{k}-\boldsymbol{U m})]^{1 / 2}-\left[k^{2}-\boldsymbol{k}^{T} \boldsymbol{k}\right]^{1 / 2}\right\}\right) \\
= & \frac{4 \pi^{2}}{|\operatorname{det} \boldsymbol{V}|} \sum_{\boldsymbol{m}} A(\boldsymbol{k}-\boldsymbol{U m}) \\
& \times \exp (j \phi(\boldsymbol{k}-\boldsymbol{U m})) \exp (-j \phi(\boldsymbol{k}))
\end{aligned}
$$

where

$$
\phi(\boldsymbol{k})=\left[k^{2}-(\boldsymbol{k})^{T}(\boldsymbol{k})\right]^{1 / 2} Z .
$$

Finally, we obtain the desired solution as

$$
\psi_{R}(\boldsymbol{x})=\mathcal{F}^{-1}\left\{\Psi_{R}(\boldsymbol{k})\right\}=\frac{1}{4 \pi^{2}} \int \Psi_{R}(\boldsymbol{k}) \exp \left[j \boldsymbol{k}^{T} \boldsymbol{x}\right] \mathrm{d} \boldsymbol{k} .
$$

Therefore, the exact effect of reconstruction from a sampled diffraction pattern is obtained as given in Eqs. (21) and (23). It is not difficult to interpret this result. Let us define $\psi_{R, \boldsymbol{m}}(\boldsymbol{x})$ as

$$
\begin{aligned}
\psi_{R, \boldsymbol{m}}(\boldsymbol{x}) \triangleq & \mathcal{F}^{-1}\left\{\frac { 4 \pi ^ { 2 } } { | \operatorname { d e t } \boldsymbol { V } | } A ( \boldsymbol { k } - \boldsymbol { U } \boldsymbol { m } ) \operatorname { e x p } \left(j Z \left\{\left[k^{2}-(\boldsymbol{k}-\boldsymbol{U} \boldsymbol{m})^{T}\right.\right.\right.\right. \\
& \left.\left.\left.\times(\boldsymbol{k}-\boldsymbol{U} \boldsymbol{m})]^{1 / 2}-\left[k^{2}-\boldsymbol{k}^{T} \boldsymbol{k}\right]^{1 / 2}\right\}\right)\right\}
\end{aligned}
$$

so that

$$
\psi_{R}(\boldsymbol{x})=\sum_{\boldsymbol{m}} \psi_{R, \boldsymbol{m}}(\boldsymbol{x})
$$

as a consequence of Eqs. (21) and (23). First of all, $\psi_{R, \boldsymbol{o}}(\boldsymbol{x})$ is the perfect reconstruction. And, since each $\psi_{R, \boldsymbol{m}}(\boldsymbol{x})$ term is associated with a translated function $A(\boldsymbol{k}-\boldsymbol{U} \boldsymbol{m})$ in the Fourier domain, these terms are modulated versions of $\psi_{R, \boldsymbol{0}}(\boldsymbol{x})$ by $\exp \left[j(\boldsymbol{U} \boldsymbol{m})^{T} \boldsymbol{x}\right]$. Please note that this modulation effect is the same as in the Fresnel case. ${ }^{8}$ However, in contrast to the Fresnel case, frequency components of $\psi_{R, \boldsymbol{m}}(\boldsymbol{x})$ shift by a different amount in space due to the nonlinear phase, $\phi_{\boldsymbol{m}}(\boldsymbol{k}) \triangleq \phi(\boldsymbol{k}-\boldsymbol{U} \boldsymbol{m})-\phi(\boldsymbol{k})$, resulting in a dispersion. We can look further at the form of the dispersion: a Taylor-series expansion of the terms in square root yields the constant, linear, and the higher-order terms of $\phi(\boldsymbol{k})$ as

$$
\phi(\boldsymbol{k})=k Z-\frac{1}{2 k} \boldsymbol{k}^{T} \boldsymbol{k} Z+\text { higher-order terms } .
$$

Therefore,

$$
\begin{aligned}
\phi_{\boldsymbol{m}}(\boldsymbol{k})= & \phi(\boldsymbol{k}-\boldsymbol{U m})-\phi(\boldsymbol{k}) \\
= & -\frac{1}{k} Z(\boldsymbol{U m})^{T} \boldsymbol{k}+\frac{1}{2 k} Z(\boldsymbol{U m})^{T}(\boldsymbol{U m}) \\
& + \text { higher-order terms. }
\end{aligned}
$$

The first term of Eq. (27) is the linear phase term in the Fourier domain and therefore corresponds to a translation in space; the location of the 2-D translation is $-(Z / k) \boldsymbol{U m}$. The second term is just a constant, and therefore it corresponds to a multiplication of the field by a complex constant. Higher-order terms create the dispersion around the location of translation. Please note that the linear term is more dominant, and therefore the higher-order terms are less significant for those $\boldsymbol{m}$ closer to $\boldsymbol{0}$. Therefore, we conclude that the dispersion due to the nonlinear phase is stronger as $\boldsymbol{m}$ increases.

Thus we reach the final result: the reconstruction from a sampled diffraction pattern yields a superposition of terms corresponding to the exact reconstruction and its modulated, translated, and dispersed versions; when the translations are far away, the modulation is higher in frequency, and the dispersion is stronger, yielding more spread-out versions of the original. Depending on the sampling rate and the spatial structure of the object, the dispersion for the components with farther translations 
(larger $\boldsymbol{m}$ ) may be so strong (spreads the object too much) that the translated objects are no longer recognizable.

It is interesting to note that, by definition, the Fresnel case has only up to the linear term in the Taylor-series expansion above. Therefore, there is no dispersion but only modulated and translated replicas of the perfect reconstruction. ${ }^{8}$ The Fresnel case is an approximation to diffraction, whereas the Rayleigh-Sommerfeld case, as also shown in this paper, is exact for the scalar case. The effects of sampling for the Fresnel case given by $\mathrm{Onural}^{8}$ is valid only if the diffraction field, which is then sampled, can be approximated by a paraxial propagation and the subsequent reconstruction is carried out digitally, again by simulating the Fresnel transform. However, if the diffraction field does not satisfy the Fresnel conditions or if the reconstruction after the sampling is carried out by optical means or by digital computation simulating the Rayleigh-Sommerfeld diffraction, there is dispersion, as explained above.

It is important to note that the dispersed and translated components in the reconstruction may be an advantage in some applications: it reduces the unwanted effects of sampling by washing out the strongly visible undesired components. However, the energy in those components does not get smaller but just spreads out, creating small but far-extending deterministic noiselike components that corrupt the desired perfect reconstruction component. Therefore, it may not be possible to completely eliminate those higher-order term components by windowing as in the Fresnel case. ${ }^{8}$ A simulated example, for a rectangular sampling grid, is illustrated in Figs. 3 through 6. The locations of translations, the modulation of the translated patterns, and the dispersion are clearly visible. Furthermore, the increase of the dispersion, as the order of diffractions increases, is also visible.

It should also be noted that the range of the summation index $\boldsymbol{m}$ is limited to those $\boldsymbol{m}$ that result in propagating waves, since we have excluded the evanescent components in our discussions.

\section{PLANE-WAVE DECOMPOSITION EQUIVALENT OF SAMPLING}

The exact analytical solution of the effect of sampling of the scalar diffraction is given in Section 3, together with interpretations of the results. To give a better insight to the mathematical analysis provided in Section 3, a physical interpretation may be useful. Such a physical interpretation is provided here in this section.

It can be assumed that the 2-D function $p(\boldsymbol{x})$ defined by Eq. (17) is obtained by intersecting a hypothetical 3-D monochromatic field:

$$
\begin{aligned}
p(\mathbf{x}) & =\sum_{\boldsymbol{m}} p_{\boldsymbol{m}}(\mathbf{x}) \\
& =\frac{1}{|\operatorname{det} \mathbf{V}|} \sum_{\boldsymbol{m}} \exp \left[j\left(-k_{x, \boldsymbol{m}} x-k_{y, \boldsymbol{m}} y \pm k_{z, \boldsymbol{m}}(z-Z)\right)\right],
\end{aligned}
$$

where, as usual, $k_{z, \boldsymbol{m}}=\left(k^{2}-k_{x, \boldsymbol{m}}^{2}-k_{y, \boldsymbol{m}}^{2}\right)^{1 / 2}$. We call this bundle of discrete angle 3-D plane waves the 3-D sampling waves: the illumination of the diffraction mask $\psi_{Z}(\boldsymbol{x})$ by this bundle, $p(\mathbf{x})$, of discrete angle plane waves samples the 2-D field by a regular 2-D lattice at the $z=Z$ plane. Note that, while the $+k_{z}$ choice corresponds to forward propagation of the sampling plane waves, $-k_{x}$ corresponds to backward propagation. As usual, complex $\mathbf{k}_{\boldsymbol{m}}$ involves evanescent waves due to imaginary $k_{z, m}$; the evanescent waves correspond to higher-frequency components, $\left(k_{x}, k_{y}\right)$, of the impulsive sampling lattice.

Therefore, instead of the single illuminating plane wave incident perpendicularly on the diffraction mask for the purpose of reconstruction, now we are illuminating the mask with a collection of many plane waves that superpose to form the sampling lattice at $z=Z$ as given by Eq. (28). The superposition of Eq. (28) includes both propagating plane waves (corresponding to lowerfrequency components of the impulsive sampling lattice) and evanescent plane waves (higher-frequency components). Since evanescent components are quickly attenuated as we go away from the sampling impulses, they do not affect the field at larger distances from the sampling plane. Therefore, we ignore the evanescent components.

Let us start with the analysis of the reconstruction by a single oblique plane-wave illumination of the diffraction mask in the reverse direction. Therefore, the incident oblique wave,

$$
\begin{aligned}
p_{\boldsymbol{m}}(\mathbf{x})= & \frac{1}{|\operatorname{det} \boldsymbol{V}|} \exp \left\{j \left[k_{x, \boldsymbol{m}} x+k_{y, \boldsymbol{m}} y\right.\right. \\
& \left.\left.+\left(k^{2}-k_{x, \boldsymbol{m}}^{2}-k_{y, \boldsymbol{m}}^{2}\right)^{1 / 2}(z-Z)\right]\right\},
\end{aligned}
$$

modulates the mask to yield the field at the $z=Z$ plane as

$$
\begin{aligned}
\psi_{Z}(\boldsymbol{x}) p_{\boldsymbol{m}}(\boldsymbol{x})= & \psi_{Z}(\boldsymbol{x}) \frac{1}{|\operatorname{det} \boldsymbol{V}|} \exp \left\{j\left[k_{x, \boldsymbol{m}} x+k_{y, \boldsymbol{m}} y\right]\right\} \\
= & \frac{1}{|\operatorname{det} \boldsymbol{V}|} \iint A\left(k_{x}, k_{y}\right) \\
& \times \exp \left(j\left(k^{2}-k_{x}^{2}-k_{y}^{2}\right)^{1 / 2} Z\right) \exp \left(j \left\{\left(k_{x}+k_{x, \boldsymbol{m}}\right) x\right.\right. \\
& \left.\left.+\left(k_{y}+k_{y, \boldsymbol{m}}\right) y\right\}\right) \mathrm{d} k_{x} \mathrm{~d} k_{y} .
\end{aligned}
$$

The reconstructed $3-\mathrm{D}$ field, as a consequence of the above oblique plane-wave illumination, is

$$
\begin{aligned}
\psi_{\boldsymbol{m}}(\mathbf{x})= & \frac{1}{|\operatorname{det} \boldsymbol{V}|} \iint A\left(k_{x}, k_{y}\right) \exp \left(j\left(k^{2}-k_{x}^{2}-k_{y}^{2}\right)^{1 / 2} Z\right) \\
& \times \exp \left(j \left\{\left(k_{x}+k_{x, \boldsymbol{m}}\right) x+\left(k_{y}+k_{y, \boldsymbol{m}}\right) y\right.\right. \\
& \left.\left.+\left[k^{2}-\left(k_{x}+k_{x, \boldsymbol{m}}\right)^{2}-\left(k_{y}+k_{y, \boldsymbol{m}}\right)^{2}\right]^{1 / 2}(z-Z)\right\}\right) \mathrm{d} k_{x} \mathrm{~d} k_{y} .
\end{aligned}
$$

Thus at $z=0$, the reconstructed 2-D field, $\psi_{R, \boldsymbol{m}}(\boldsymbol{x})$, becomes

$$
\begin{aligned}
\psi_{R, \boldsymbol{m}}(\boldsymbol{x})= & \frac{1}{|\operatorname{det} \mathbf{V}|} \iint A\left(k_{x}, k_{y}\right) \cdot \exp \left(j \left\{\left(k^{2}-k_{x}^{2}-k_{y}^{2}\right)^{1 / 2}\right.\right. \\
& \left.\left.-\left[k^{2}-\left(k_{x}+k_{x, \boldsymbol{m}}\right)^{2}-\left(k_{y}+k_{y, \boldsymbol{m}}\right)^{2}\right]^{1 / 2}\right\} Z\right) \\
& \cdot \exp \left(j\left[\left(k_{x}+k_{x, \boldsymbol{m}}\right) x+\left(k_{y}+k_{y, \boldsymbol{m}}\right) y\right]\right) \mathrm{d} k_{x} \mathrm{~d} k_{y} .
\end{aligned}
$$




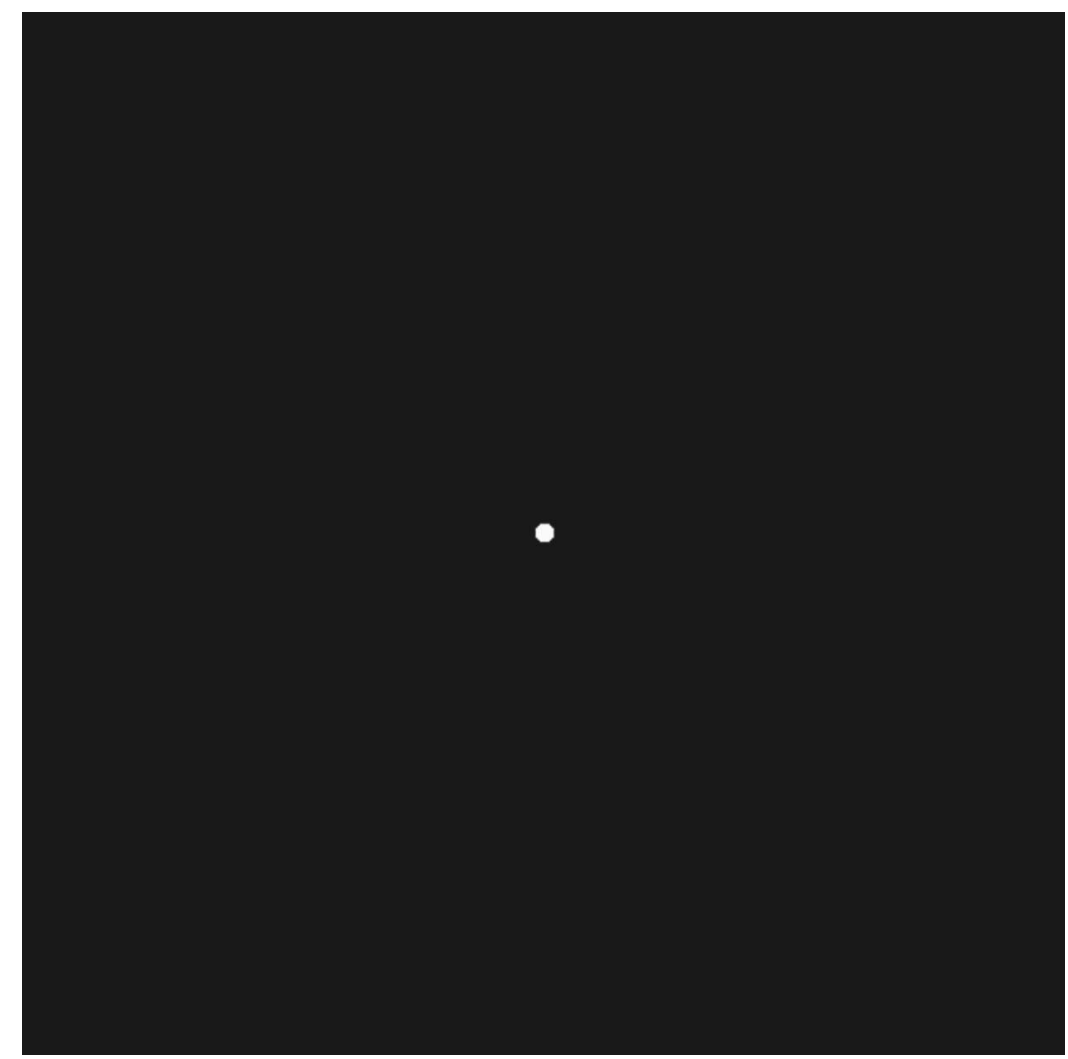

Fig. 1. Circular aperture.

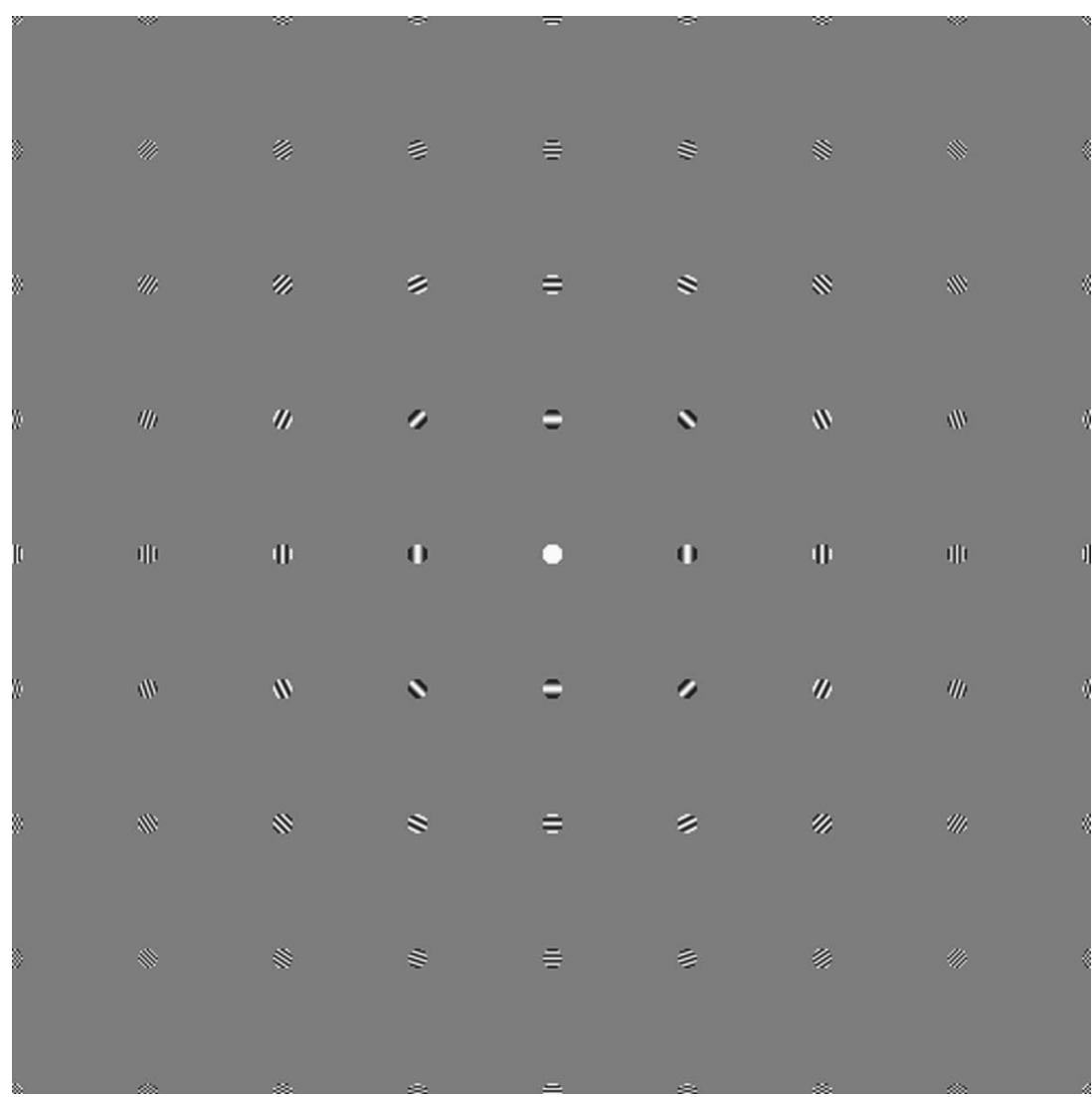

Fig. 2. Fresnel reconstruction from the sampled Fresnel diffraction pattern of the object given in Fig. 1. The real part of the field is shown. 


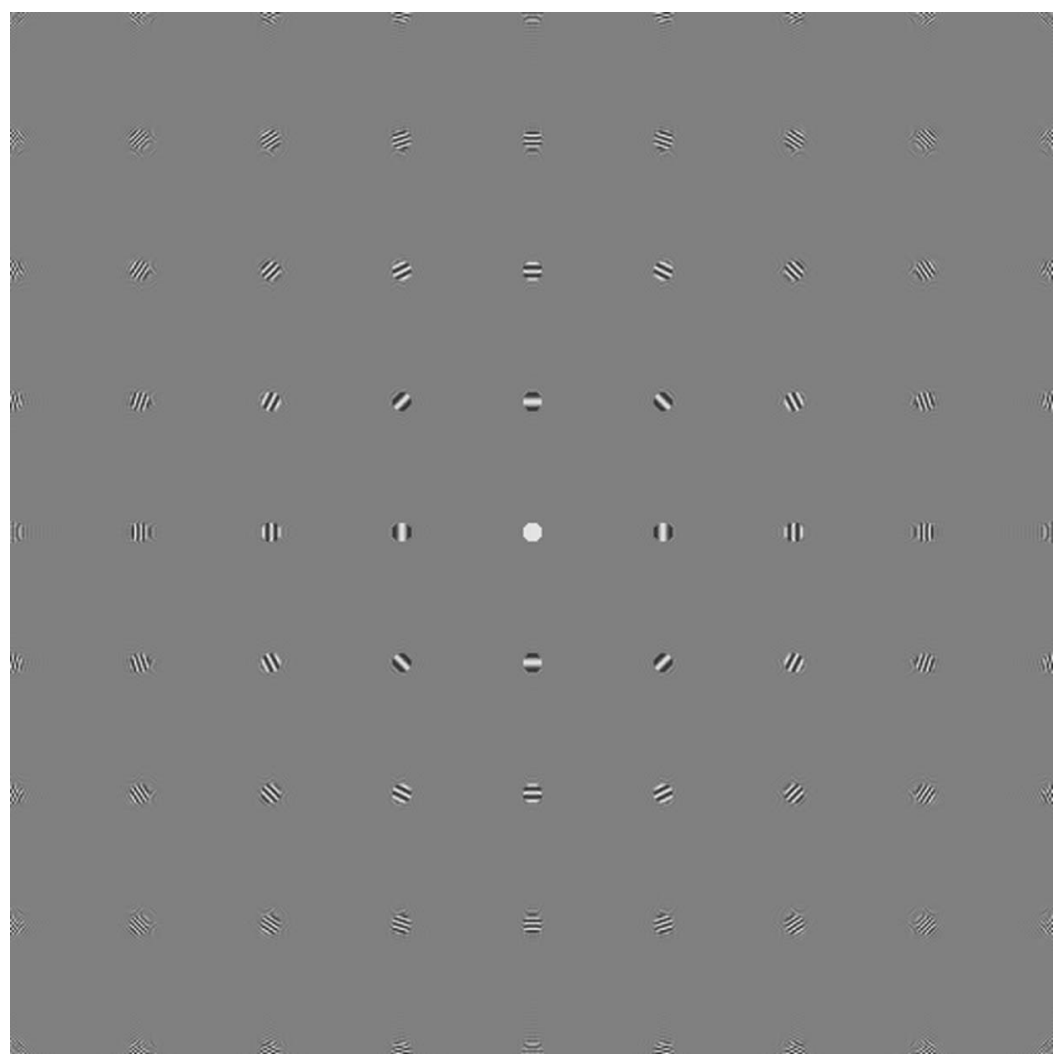

Fig. 3. Rayleigh-Sommerfeld reconstruction from the sampled Rayleigh-Sommerfeld diffraction pattern of the object given in Fig. 1. The distance is large, and therefore the pattern is similar to the Fresnel case. The real part of the field is shown.

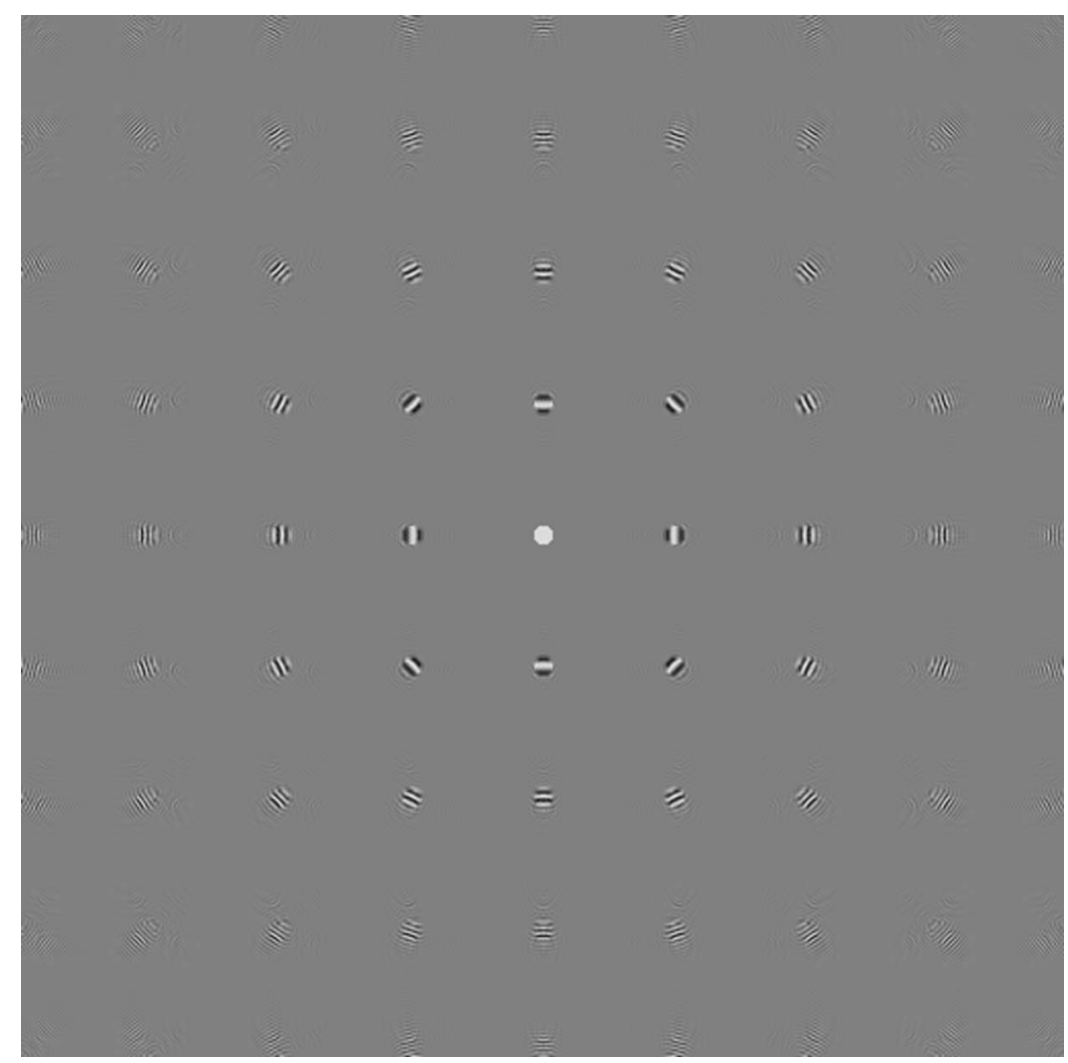

Fig. 4. Rayleigh-Sommerfeld reconstruction from the sampled Rayleigh-Sommerfeld diffraction pattern of the object given in Fig. 1. The distance is smaller than that of Fig. 3, and therefore the discussed dispersion effects are more visible. The real part of the field is shown. 


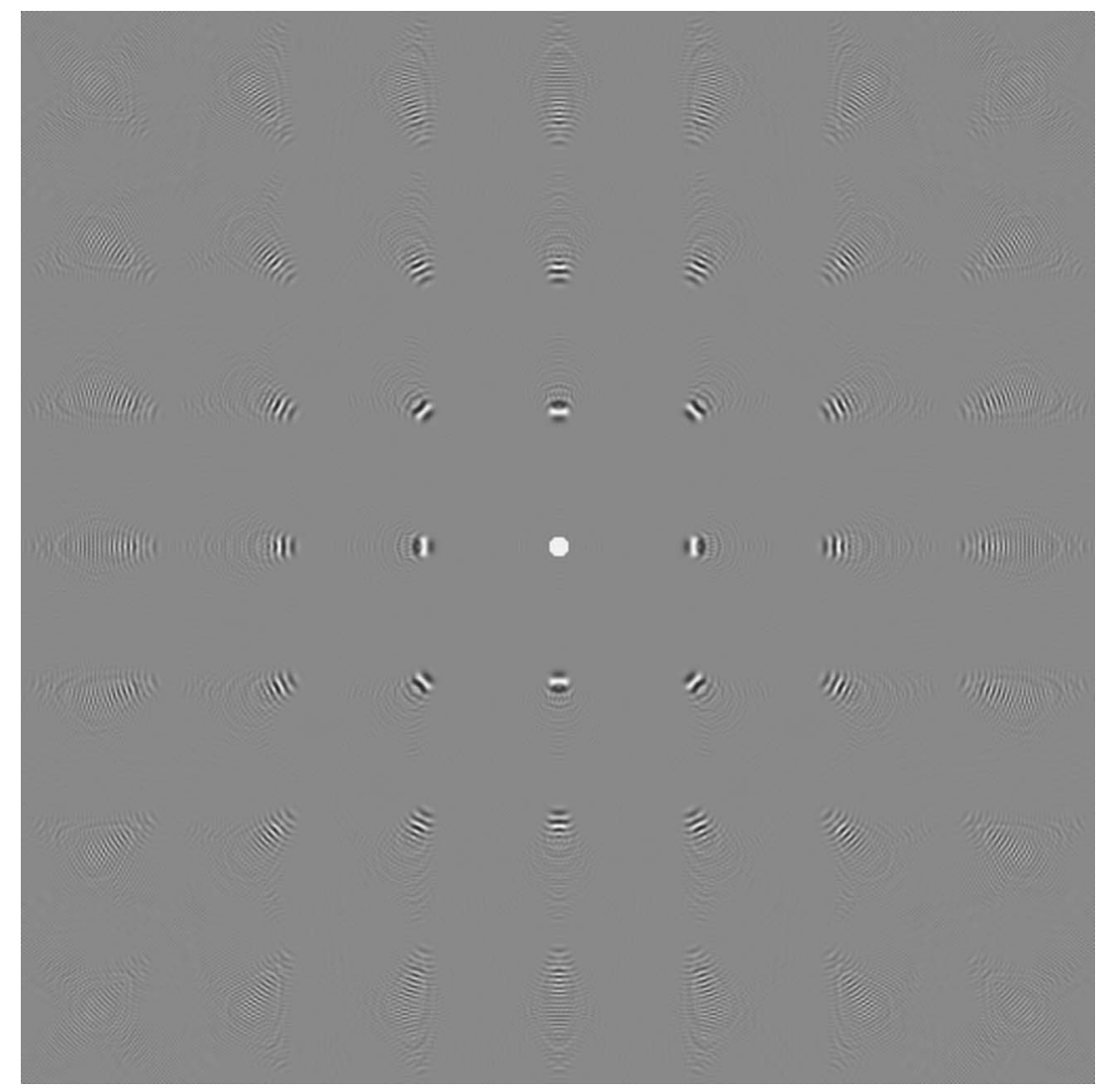

Fig. 5. Same simulation as in Fig. 4 but with a smaller diffraction distance.

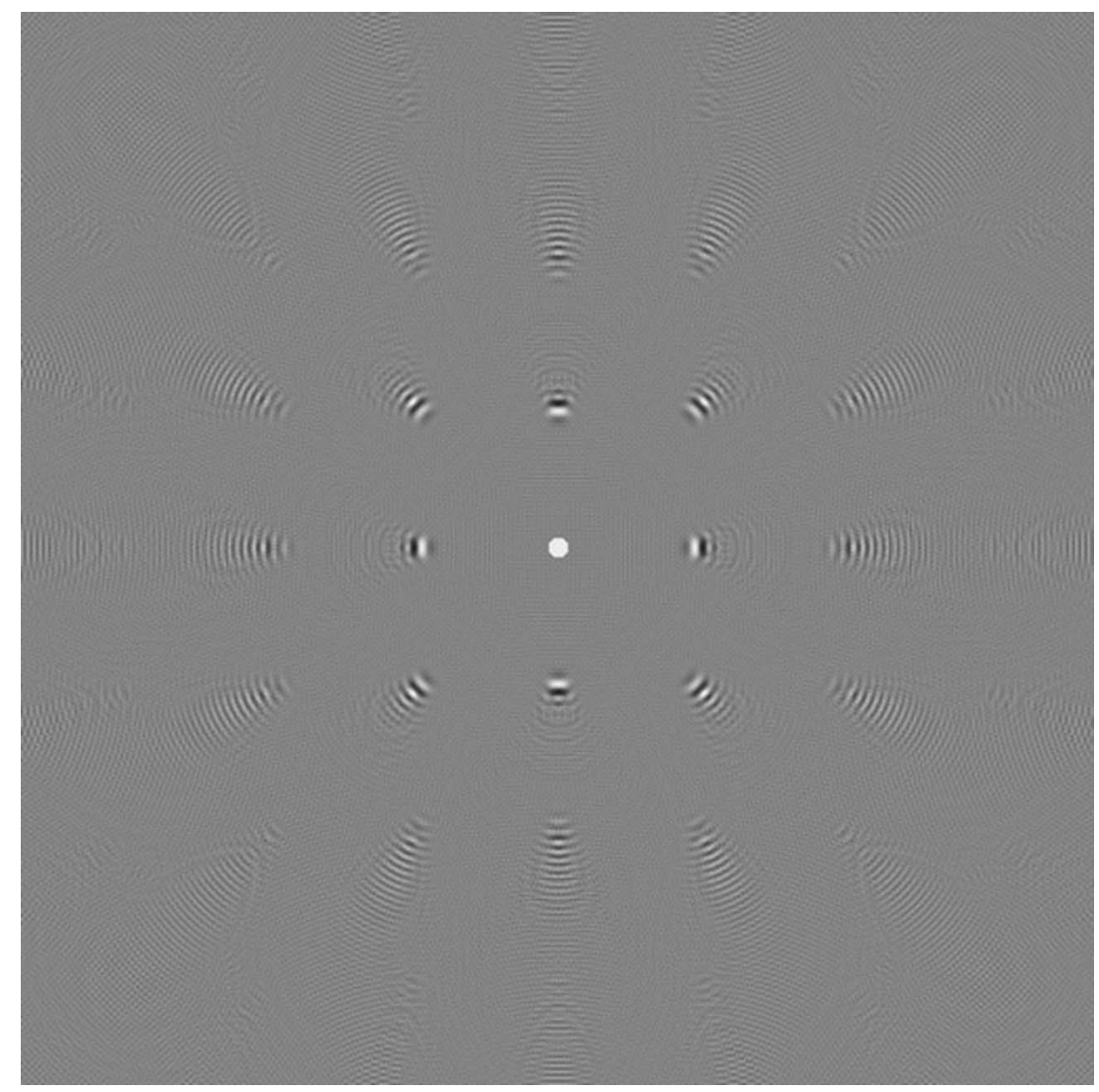

Fig. 6. Same simulation as in Fig. 5 but with a smaller diffraction distance. 


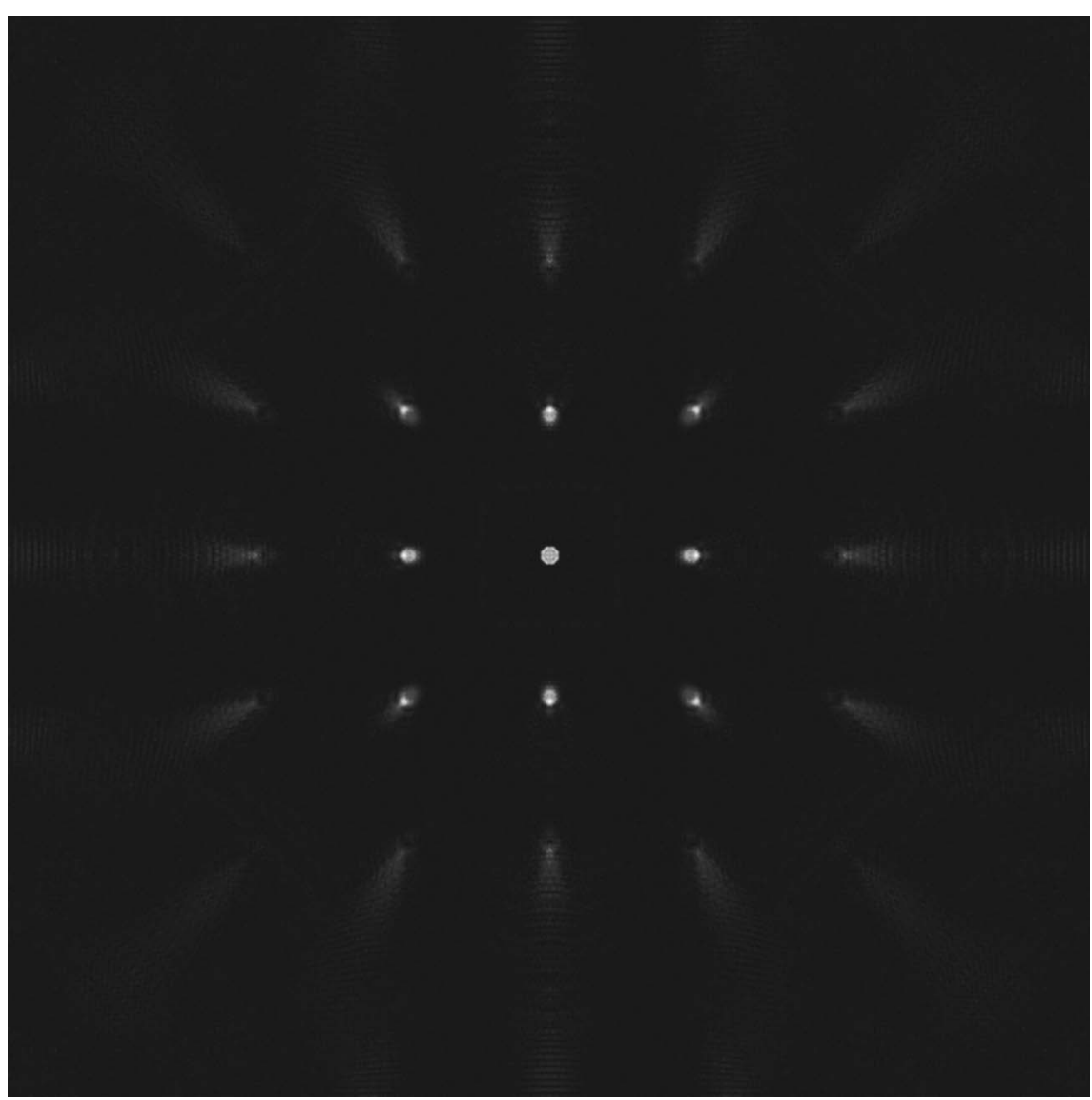

Fig. 7. Intensity pattern of the field whose real part is shown in Fig. 6 .

A change of variables, $k_{x}^{\prime}=k_{x}+k_{x, \boldsymbol{m}}$ and $k_{y}^{\prime}=k_{y}+k_{y, \boldsymbol{m}}$, yields

$$
\begin{aligned}
\psi_{R}(\boldsymbol{x})= & \frac{1}{|\operatorname{det} \mathbf{V}|} \iint A\left(k_{x}^{\prime}-k_{x, \boldsymbol{m}}, k_{y}^{\prime}-k_{y, \boldsymbol{m}}\right) \cdot \exp \left(j \left\{\left(k^{2}\right.\right.\right. \\
& \left.\left.\left.-\left(k_{x}^{\prime}-k_{x, \boldsymbol{m}}\right)^{2}-\left(k_{y}^{\prime}-k_{y, \boldsymbol{m}}\right)^{2}\right)^{1 / 2}-\left[k^{2}-{k_{x}^{\prime}}^{2}-k_{y}^{\prime 2}\right]^{1 / 2}\right\} Z\right) \\
& \cdot \exp \left(j\left(k_{x}^{\prime} x+k_{y}^{\prime} y\right)\right) \mathrm{d} k_{x}^{\prime} \mathrm{d} k_{y}^{\prime} .
\end{aligned}
$$

Therefore, we conclude that the reconstructed field from the sampled diffraction pattern is the inverse Fourier transform from the $\left(k_{x}^{\prime}, k_{y}^{\prime}\right)$ domain to $(x, y)$ :

$$
\begin{aligned}
\psi_{R}(\boldsymbol{x})= & \frac{1}{|\operatorname{det} \mathbf{V}|} \mathcal{F}^{-1}\left\{A\left(k_{x}^{\prime}-k_{x, \boldsymbol{m}}, k_{y}^{\prime}-k_{y, \boldsymbol{m}}\right)\right. \\
& \times \exp \left(j \left\{\left[k^{2}-\left(k_{x}^{\prime}-k_{x, \boldsymbol{m}}\right)^{2}-\left(k_{y}^{\prime}-k_{y, \boldsymbol{m}}\right)^{2}\right]^{1 / 2}\right.\right. \\
& \left.\left.\left.-\left[k^{2}-k_{x}^{\prime 2}-k_{y}^{\prime 2}\right]^{1 / 2}\right\} Z\right)\right\} .
\end{aligned}
$$

Repeating for each such illuminating oblique plane-wave component of Eq. (28) and summing over all such reconstructions, we get

$$
\psi_{R}(\boldsymbol{x})=\sum_{\boldsymbol{m}} \psi_{R, \boldsymbol{m}}(\boldsymbol{x}),
$$

which is the same as the previously derived Eq. (23).

Provided digitally simulated figures demonstrate the effects interpreted above. A test object, which is a circular aperture, is shown in Fig. 1. A Rayleigh-Sommerfeld diffraction pattern is computed by digital simulation. Afterward, the diffraction pattern is sampled by keeping one out of eight pixels in each direction (one out of 64 samples are kept in a rectangular area); the rest of the samples (63 of them) are replaced by zeros. The sampled pattern is then numerically reconstructed by simulating RayleighSommerfeld diffraction. Simulations are repeated for different effective $Z$ values. For comparison, a Fresnel case is also presented. Figure 2 is the digitally simulated Fresnel reconstruction from the digitally computed and sampled Fresnel diffraction pattern. Figures 3-6 are digitally simulated Rayleigh-Sommerfeld reconstructions at $Z$ effectively different from the digitally computed and sampled Rayleigh-Sommerfeld diffraction pattern. Figure 3 shows the largest distance (closer to Fresnel range), and the distance gets smaller in Figs. 4-6. In each case, the real part of the field is demonstrated. Sharp boundaries of the shifted and modulated replicas of the perfect reconstruction (the circle at the center) in the Fresnel case is typical. However, the higher-order diffraction components are spread out in the Rayleigh-Sommerfeld case. In agreement with the theoretical analysis and interpretation above, the spread is stronger as the diffraction order increases. Similarly, the spread is more visible as the distance $Z$ gets smaller, and thus the exact (RayleighSommerfeld) diffraction deviates more from the Fresnel case. Figure 7 shows the intensity image of the pattern whose real part is illustrated in Fig. 6; as seen from this figure, the reconstruction is good even if 63 out of 64 samples are zeroed out due to sampling.

\section{CONCLUSIONS}

An exact analytical solution to find the effects of sampling of the scalar diffraction field on the reconstruction is de- 
rived. The analysis of this solution indicates that the reconstruction from the sampled diffraction pattern contains an exact reconstruction of the original object (within a gain factor); however, this exact reconstruction is contaminated by the superposition of a number of distorted and translated versions of the reconstructed original. The distortion includes a modulation by a complex sinusoid; but a more important distortion is the dispersion of these translated components. The dispersion gets stronger for far-away components. This dispersion may or may not be desirable: It washes out the translated components by spreading them out and thus makes them less visible, but it also creates small but spread-out deterministic noise terms that may overlap with the desired reconstruction component.

A comparison of the effects of sampling for the Rayleigh-Sommerfeld (plane-wave decomposition) case with the Fresnel case ${ }^{8}$ indicates that both cases have the expected higher diffraction orders translated to the same locations and modulated by the same complex sinusoidals, but there is no dispersion in the Fresnel case.

It is also shown that, for the scalar case, the effect of sampling may be interpreted as the illumination of the mask by a bundle of different angle incident plane waves.

\section{ACKNOWLEDGMENTS}

This work is supported by the European Commission within FP6 under grant 511568 with the acronym 3DTV. The author thanks Gökhan Bora Esmer for preparing the pictures used in the figures.

The author can be reached by e-mail at onural@ee.bilkent.edu.tr.

\section{REFERENCES}

1. L. Onural and P. D. Scott, "Digital decoding of in-line holograms," Opt. Eng. 26, 1124-1132 (1987).

2. J. H. Milgram and W. Li, "Computational reconstruction of images from holograms," Appl. Opt. 41, 853-864 (2002)

3. U. Schnars and W. P. O. Juptner, "Digital recording and numerical reconstruction of holograms," Meas. Sci. Technol. 13, R85-R101 (2002).

4. C. Wagner, S. Seebacher, W. Osten, and W. Jüptner, "Digital recording and numerical reconstruction of lensless
Fourier holograms in optical metrology," Appl. Opt. 38, 4812-4820 (1999).

5. Y. Takaki and H. Ohzu, "Fast numerical reconstruction technique for high-resolution hybrid holographic microscopy,” Appl. Opt. 38, 2204-2211 (1999).

6. Y. Zhang, G. Pedrini, W. Osten, and H. J. Tiziani, "Image reconstruction for in-line holography with Yang-Gu algorithm," Appl. Opt. 42, 6452-6457 (2003).

7. L. Yu, Y. An, and L. Cai, "Numerical reconstruction of digital holograms with variable viewing angles," Opt. Express 10, 1250-1257 (2002).

8. L. Onural, "Sampling of the diffraction field," Appl. Opt. 39, 5929-5935 (2000).

9. L. Onural, "Some mathematical properties of the uniformly sampled quadratic phase function and associated issues in Fresnel diffraction simulations," Opt. Eng. 43, 2557-2563 (2004).

10. A. Stern and B. Javidi, "Analysis of practical sampling and reconstruction from Fresnel fields," Opt. Eng. 43, 239-250 (2004).

11. F. S. Roux, "Complex-valued Fresnel-transform sampling," Appl. Opt. 34, 3128-3135 (1995).

12. A. Stern and B. Javidi, "Improved resolution digital holography using generalized sampling theorem for locally band-limited fields," J. Opt. Soc. Am. A 23, 1227-1235 (2006).

13. F. Gori, "Fresnel transform and sampling theorem," Opt. Eng. 39, 293-297 (1981).

14. J. M. Coupland, "Holographic particle velocimetry: signal recovery from under-sampled CCD data," Meas. Sci. Technol. 15, 711-717 (2004).

15. J. W. Goodman, Introduction to Fourier Optics, 2nd ed. (McGraw-Hill, 1996).

16. M. Born and E. Wolf, Principles of Optics, 7th ed. (Cambridge U. Press, 2003).

17. P. C. Clemmow, The Plane Wave Spectrum Representation of Electromagnetic Fields (Oxford U. Press and IEEE Press, reissue, 1996).

18. M. Ayatollahi and S. Safavi-Naeini, "A new representation for the Green's function of multilayer media based on plane wave expansion," IEEE Trans. Antennas Propag. 52, 1548-1557 (2004).

19. L. Onural, "Impulse functions over curves and surfaces and their applications to diffraction," J. Math. Anal. Appl. 322, 18-27 (2006).

20. L. Onural, "Projection-slice theorem as a tool for mathematical representation of diffraction," IEEE Signal Process. Lett. 14, 43-46 (2007).

21. G. C. Sherman, "Application of the convolution theorem to Rayleigh's integral formulas," J. Opt. Soc. Am. 57, 546-547 (1967).

22. É. Lalor, "Conditions for validity of the angular spectrum of plane waves,” J. Opt. Soc. Am. 58, 1235-1237 (1968).

23. D. E. Dudgeon and R. M. Mersereau, Multidimensional Signal Processing (Prentice Hall, 1984). 